$\mathbb{T}$ periodica polytechnica

\author{
Social and Management Sciences \\ $18 / 2(2010) 63$ 67 \\ doi: 10.3311/pp.so.2010-2.02 \\ web: http://www.pp.bme.hu/so \\ (c) Periodica Polytechnica 2010
}

RESEARCH ARTICLE

\section{Influence of personality on Teamwork behaviour and communication}

Márta Juhász

Received 2010-11-08

\begin{abstract}
Job characteristics of the operator teams of the Nuclear Power Plant are complex and highly controlled in which there are considerable demands and pressures to behaviour conformity and a person is restricted in the range of own behaviour. Thus, individual differences in personality characteristics are more likely to influence the specific behaviour a person adopts. This type of environment determines and regulates the communication flow among team members that consist of quantity and quality information exchanges. All these circumstances lead our focus on analysing the relationship between the employees' communication and observable behaviour and their personality traits.
\end{abstract}

We video registered 17 operator teams $(N=90)$ in a Simulator Centre of a Hungarian Nuclear Power Plant and analysed the correlation between the team input (operator personnel's personality traits) and team process (communication hidden patterns, traceable teamwork-oriented social skills and taskoriented professional skills), and ultimately team output (team performance evaluated by instructors).

This study reveals some relationships between personality traits and team-oriented communication utterances. Extroversion and Openness to experience personality factors show positive correlation with Politeness and Relation communication indicators, but contrary to our expectation the Agreeableness personality factor negatively relates with these indicators. The Team-performance has several relationships with personality traits. First of all Professional knowledge and Coordination behaviour markers show correlations with Neuroticism and Conscientiousness personality factors. Team-performance as an output of the team process is directly influenced by the Conscientiousness and the Extraversion personality factors.

\section{Keywords}

personality · Five Factor Model · team performance · communication utterances $\cdot$ behaviour markers

\section{Márta Juhász}

Department of Ergonomics and Psychology, BME, 1111 Budapest, Egry J. u. 1., Hungary

e-mail: juhaszm@erg.bme.hu

\section{Teamwork in high risk environment}

Numerous organisations tend to require effective professional teamwork in a high risk environment because expert teams have deep professional knowledge and are supposed to have fewer personal problems and conflicts between the members. Team members studied a lot in order to belong to a certain team, so everybody in the team is considered to be an expert in their own professional fields. They cooperate in order to achieve a given task following and complying with the rules. High risk environments mean that certain failures of teamwork in complex organizations can lead to dramatic effects. That is why working in this type of team can be inherently stressful for the members. The major parts of air crashes involve human errors, especially failures in teamwork. Unfortunately, professional training in general mainly focuses on technical, not interpersonal skills. These findings support the argument that technical skills are necessary but not sufficient to ensure high level of safety over time. In professional teams the personnel is strongly motivated to perform successfully and to maintain high standards of safety, so it can be assumed that they are all aware of the basic standards of professional proficiency. Operators, pilots and physicians have strong professional cultures with as many positive as negative aspects: strong motivation to do well their tasks, strong pride in their profession, sense of personal invulnerability, maintaining high individual standards, continual performance evaluation, pushing the limits of performance - "press-on", invulnerability to fatigue and other frailties, capability for individual vs. team performance. The majority of expert team members in all cultures agree that: a) their decision-making is as good in emergencies as in normal situations, b) their performance is not affected by personal problems, c) they do not make more errors under high stress, and d) true professionals leave behind the personal problems.

\section{Communication utterances in the teamwork}

Understanding the past and predicting the future behaviour of others requires the ability to imagine how other persons perceive, think, and act. Working together in a team is facilitated only if each member of the team has a theory of 
the other members' mind, ability, concepts and intents. The main channel to exchange the information is the communication. The prime task of communication is to facilitate the establishment, maintenance and modification of shared assumptions about each other's minds. The maintenance of communication means adding information, modifying it in a way that is obvious to all members of the team. If such modifications are accepted, the modification becomes part of a team's common ground, and the team members will act accordingly. In this way, the formed common ground constitutes the basis of a shared mental model that gives coherence to the action of a team. The common ground includes information. The understanding of this information in communication is usually indicated by acknowledgements like $o k, m m-h m m$, and gestures like nodding. This kind of communication utterances may be factual claims, questions, commands, common goals, objectives. The most important device by which common grounds can be maintained and changed is the language. From this perspective it is necessary to understand numerous important properties of language. One theoretical model for that is 'dynamic interpretation', which understands the meaning of utterances as their propensity to change common grounds [19, 20,23].

The NASA researchers analyzed the causes of airport accidents and incidents between 1968 and 1976, and concluded that pilot error was more likely correlated to failures in team communication and coordination than deficiencies in technical proficiency $[\overline{10}]$.

"While language is not only used for communication (think of thinking out aloud, as in organizing one's thoughts), communication is one of the prime uses of language. And while communication is possible without the use of language (think of gestures, signs, pictures, alarm sounds), communication is certainly facilitated by language. Communication in turn is essential for the coordination of joint actions in groups or teams." (in: Krifka, 2004. [20] 1.pp)

Heimlich "Good communication" is one of the main aspects of the team work especially under high risk environment because the communication is an important part of the interaction. Good communication is crucial for excellent crews, and communication errors reflect deficiencies of cooperation in general. In order to share the information and form a shared mental model or develop a common understanding of the nature of events among team members, communication is critical. Crew performance is more closely associated with the quality of crew communication than with the technical proficiency of an individual pilot [34].

The relationship of team performance and communication utterances was examined by several researchers [37]; [38]; [20]; [12].

Most studies are related to the relationship between communication problems and the cognitive load of the operators in the team. In line with this, one of the most famous studies, the Lin- guistic Factors Project [35], [23] investigates the fine structure of verbal communication of cockpit crews. In this project properties of verbal communication are identified that correlate with the task load and performance of crews that are faced with complex and potentially dangerous tasks. In their analysis it is pointed out that members of well-performing crews more often refer to their crew by pronouns like we, and that communication density increases with high work load.

Speech Act Theory, as initiated by John Austin and John Searle [33] started observing socially relevant acts of the speaker like commands, permissions, promises, apologies, insults, or even more specific ones like hiring a person or declaring an emergency. Speech acts can be part of a more complex communicative interaction, directly relating to preceding or subsequent speech acts, as with questions that request an answer or acknowledgements that express understanding of agreement with a previous utterance. Traditionally, the speech act theory is characterized by a more deductive, rather than empirical, research methodology. But there are a number empirical studies, e.g., class room communication analysis [11], or the analysis of mitigation and reinforcement, that show that the speech act theory can be fruitfully applied in the analysis of real communication events.

13 distinct types have been developed by means of cockpit speech analysing, in particular status reports, which report on the current state of equipment, weather, location; reports of action, in which the speaker gives a report of his own actions; reports of reports, which rephrase information expressed before; prognoses about the likely future course of events; diagnoses, which are concerned with the likely cause of past events; commands and permissions, by which one crew member can directly influence the actions of others; complies, which verbalize actions that are performed to carry out a command; reports of intention, which express the intention to act in a certain way; expressive, which express an emotion; and three types of acknowledging speech acts: simple acknowledgments, affirmations and rephrases.

Conversation Analysis, as initiated by Harvey Sacks [31], [16] has developed ways of transcribing conversation in minute details and of analyzing it in objective ways without paying attention to the participants' intentions. Phenomena like the introduction, continuation and uptake of topics of conversation, the negotiation of turn taking between participants, the methods of repairing utterances, and in general the sequencing of contributions are crucial for the conversation analyst. Conversation analysis certainly leads to deep insights into conversation and it is very useful to illustrate "good" and "bad" communication for the instruction of expert teams.

Pennebaker (1999) has shown that linguistic styles can be considered individual difference markers, i.e., individuals appear to have a distinct language use fingerprint which is relatively stable across time and situations [29]; [32]. His lab has identified language dimensions to be internally consistent, and 
modestly correlated to objective and self-reported health and performance measures at rates comparable to or greater than traditional trait markers of personality such as the Big Five [24].

According to Krifka, Martens and Schwarz study (2003), more 'politeness' markers were used by poorly performing crews. Powerful participants, or participants that are familiar with each other, need not resort to face-saving strategies in the same way as less powerful or familiar participants do. The general need of face-saving make people try to save the others' faces by avoiding face-threatening acts, or by mitigating them if they cannot be avoided. All face-preserving strategies result in expressions that are generally longer and more complex. Politeness can be interpreted by saying that the speaker explicitly shows that $\mathrm{s} / \mathrm{he}$ puts in a greater effort in the act of communication, or puts him/herself in a handicap, thus indicating the worthiness of the communicative goal that s/he wants to achieve, politeness may well be a handicap [20], [19].

\section{The Five-Factor Model and performance}

Personality is an important factor in accounting for how employees behave in teams and in the organisation. The interest in identifying personality predictors of job performance has led researchers to use the Five Factor Personality Model as an important conceptual tool. The development of the Five-Factor Model (FFM; Wiggins, 1996) [41] is an important event in the history of personality psychology because it provides taxonomy of trait terms. This is a hierarchical organization of personality traits in terms of five basic dimensions.

Research using both natural language adjectives and theoretically based personality questionnaires supports the comprehensiveness of the model and its applicability across observers and cultures [24]).

I. Neuroticism (N) The tendency to experience nervousness, tension, anxiety, emotional instability, hostility and sadness.

II. Extraversion (E) An energetic approach to the external world, including sociability, assertiveness and positive emotionality.

III. Openness to experience (O) Describes the breadth, depth, originality and complexity of an individual's mental and experiential life.

IV. Agreeableness (A) The quality of one's interpersonal interactions along a continuum from compassion and altruism to antagonism.

V. Conscientiousness (C) Persistence, organization, and motivation in goal-directed behaviours, and socially prescribed impulse control.

The predictive power of the model within the employment context has often been demonstrated [1]; [30]; [14]. Barrick and Mount's (1991) study is a 35-year view on personality-based prediction research, which identifies some salient relations between personality and work performance. They investigated the relation of the FFM personality dimensions to three job performance criteria (job proficiency, training proficiency, and personnel data) for five occupational groups (professionals, policemen, managers, sales, and skilled/semi-skilled workers). Results indicate that the Conscientiousness (C) factor shows consistent relations with all job performance criteria for all occupational groups. Extraversion (E) is a valid predictor for two occupations (managers and salesmen) involving social interaction, across various criterion types. High scores on the Extraversion (E) scale indicate a warm, engaging, positive orientation toward others. Both Openness to experience $(\mathrm{O})$ and Extraversion (E) factors are valid predictors of the training proficiency criterion (across occupations) and both factors predict a good mental ability, too. These people are open to new things, and able to learn quickly; so their supervisors very often evaluate them as the best workers.

It seems that three of the FFM (Extraversion, Agreeableness, Conscientiousness) are particularly relevant in managerial roles. Managers who score high on Extraversion (E) and Agreeableness (A) are likely better suited for the social and interpersonal demands (e.g., fostering positive work relationship, interactions with subordinates, public relations) than those who score lower on these socially relevant dimensions. The Conscientiousness (C) factor is linked to motivational processes and outcomes. Managers who score high on this factor are more likely to engage in goal-directed behaviour and to perform tasks carefully and enthusiastically than their low on Conscientiousness (C) counterparts [3]. Contrarily to expectations of the Barrick, Mount's (1991) study it is found that a certain level of Neuroticism $(\mathrm{N})$ can affect the performance at least in the professionals group.

Certain personality traits may interact with others to result in desirable, as well as undesirable, workplace behaviours. In personality research Conscientiousness $(\mathrm{C})$ has been the most consistent and universal predictor of job performance. For example, the relationship between Conscientiousness (C) and job performance is stronger for persons high on Agreeableness (A) than for those low on Agreeableness. Highly conscientious workers who lack interpersonal sensitivity may be ineffective, particularly in jobs requiring cooperative interchange with others [40]; [26].

\subsection{Teamwork and personality}

The article "Changing nature of the work" by Michael Frase (2000) suggests that nowadays the most important trend is the increased use of teamwork. Organisations have attempted to create greater flexibility and autonomy in their structures. It is thought that teams are more efficient in adapting to the organisation's dynamic environments and handle complex and variable products and processes than team members individually [7]. It is thought that teams are capable of increasing an organisation's 
adaptability to dynamic environments, are able to handle more complex and variable products and production processes, and that team members can more easily mutually adjust and coordinate their efforts. Additionally, it is believed that working in teams enhances the quality of working life and entails attractive, intrinsically motivating jobs.

Despite the renaissance of teamwork, relatively little is known about how the individual contributes to the team intragroup processes and outcomes. The dominant way of thinking about the team is the input-process-output model. The model posits that a variety of inputs combine to influence intragroup processes, which in turn affect team outputs [3].

The role of personality in team process and team performance is unarguable. Hackman [3] divided the inputs into three categories: 1) individual-level factor (e.g., team member attributes, personality, skills), 2) team-level factors (e.g., structure and size) and 3) environmental-level factors (e.g., task characteristics, level of the autonomy). Intragroup process refers to interactions that take place among the team members and include interaction patters such as conflict, efforts toward leadership and those communication patters that differentiate teams from each other (as an above-mentioned fingerprint). Each team has its own communication style depending on the environment they are working in. Team output refers to team outcomes associated with productivity, performance, as well as capability of team members to continue the work cooperatively. Hackman proposes that a comprehensive assessment of the teams should capture both current (present performance) and future team effectiveness (capability to continue working together as a unit). The most important measure of team effectiveness is the current performance assessment of the team, which is based on either supervisor ratings of team productivity or objective indicators of team quantity and quality of productivity. Another critical measure of team effectiveness is the assessment of the team's capability to continue functioning as a unit (called team viability).

Using the above-mentioned theoretical framework, our study focuses on the team-members' personality and behaviour markers ("soft" and "hard" skills) (as inputs) and their relationship with the teams' communication patters (as team-process) and the teams' performance (as output) evaluated by instructors.

In a review of Moynihan (2004) three basic theoretical perspectives explain the nature of personality effects in team performance. Universal approach: certain traits always predict teamwork process and team performance. Contingent approach: certain traits predict team performance depending on the task or organisational culture. Configurational approach: the mix of traits within a team and the fit of individual members with each other predict team performance. We can conclude that the role of personality in team process must integrate all three of these approaches.

\subsubsection{Universal approach}

Conscientiousness (C) has been examined in team performance because it is a reliable predictor of individual performance. Conscientiousness (C) has consistently been found to be positively related to task focus and positively affected the team performance, but only when both the team level conscientiousness (measured by the lowest team member) and the leader conscientiousness were high. In sum, Conscientiousness (C) has been found to be a broad predictor of team and individual performance in field and laboratory settings [3]; [21]; [4]; [39]. But it seems that in creativity tasks, for example, a brainstorming study found that when team members were allowed to discuss strategies, teams composed of highly conscientious people produced better-quality performance (in terms of feasibility), whereas teams composed of low-conscientiousness members produced a greater quantity of potential solutions. Such studies suggest tasks that require creativity may moderate the relationship between team conscientiousness and task performance. Therefore, Conscientiousness (C) may be broadly applicable across many types of tasks, but may not predict specific types of tasks that require a large degree of creativity.

The trait of Extraversion (E) has been shown to have positive effects on individual job performance for jobs requiring a high degree of social interaction [1]; [3]; [22]. Most teams require the social interaction, so it is interesting question how is the impact of Extraversion (E) in team settings. Teams higher in mean levels of Extraversion (E) received higher supervisor ratings of team performance than teams low on Extraversion (E). Teams with more extraverted members tend to be more socially cohesive and ultimately more highly evaluated by their supervisors. The degree of variance of Extraversion (E) has a curvilinear relationship to task focus and performance, suggesting that too many or too few extraverts in a team can be inefficient. In general, Extraversion (E) appears to facilitate cohesive team process, but only at moderate levels.

Teams with high mean levels of Agreeableness (A) have higher team viability, because Agreeableness is characterized by concern for a team over desires and interests. Teams of management students working on a case study analysis and presentation task, individuals high on Agreeableness (A) were more likely to be rated as cooperative team members by their peers (Barrick, $1998[3] ; 45]$. Low levels of Agreeableness (high individualism) are associated with reduced individual effort or social loafing in teams. Individuals low on Agreeableness (A) tended to be unresponsive to teammates and tended to focus on their own task performance [42,46].

Neuroticism $(\mathrm{N})$ has been identified as a detrimental variable for work-team performance. The low end of the Neuroticism (N) factor is often referred to as Emotional stability. This personality factor is positively related to team productivity. The positive affective tone of a team is related to prosocial behaviour of its members. Teams with negative affective tone (negative affectiv- 
ity or neuroticism) experienced higher rates of absenteeism. In sum Emotional stability is positively associated with cohesive team process and effective decision making.

\subsubsection{Contingent approach}

The contingent approach to personality in teams assumes that team performance is contingent on the nature of the team task or organizational culture. The implication of this perspective is that the optimal performance of a team depends on the nature of the work and the organizational culture in which it operates. These situational variables have moderating effects on the relationship between personality and team process or performance.

Some studies consider the role of moderators in the relationship between personality traits and job performance, little is known about how personality traits predict work behaviour [2]; [14]; [6]). In general psychologists analysing the personality traits predict the future behaviour in the workplace and rarely take into account those moderators that can influence prediction validity. Identification of moderators of the personality-job performance relation can increase the validity of personality measures in predicting job performance. One such moderator is the situation in which job performance takes place. For example the level of autonomy in a job performance environment moderates the relation between personality and job performance: personality-job performance correlations were higher in highly autonomous work situation than less autonomous work situation [5]. Testing the moderator role of the autonomy on personalityperformance relations revealed positive relations between both Extroversion (E) and Agreeableness (A) and the performance criteria when the autonomy was high. Negative relations are found between Agreeableness (A) and the criterion of job performance when autonomy is low. Otherwise Barrick, Mount [2] investigated the moderating role of autonomy on the relationship between the FFM dimensions and supervisor ratings of job performance. Results indicate that the Conscientiousness (C) and Extraversion (E) factors are greater for managers in jobs high in autonomy compared with those in jobs low in autonomy. The validity of Agreeableness (A) was also higher in high-autonomy jobs compared with low-autonomy ones, but the correlation was negative. These findings suggest that the degree of autonomy in the job moderates the validity of personality dimensions. These results indicate that personality-contextual performance correlations vary across situations with different expectations for performance. Personality and contextual performance behaviour is most strongly correlated when there are only weak cues and less correlated when there are strong cues.

Molleman, Nauta, Jehn examined the moderating role of the team task autonomy in the relationship between the mean level of three personality traits in a team - Conscientiousness (C), Emotional Stability $(\mathrm{N})$ and Openness to experience $(\mathrm{O})-$ and two individual outcomes: job satisfaction and learning [26]. They show that team task autonomy strengthens the relationship between Conscientiousness (C) and learning and the rela- tionship between Openness to experience $(\mathrm{O})$ and satisfaction. They conclude that team attributes and the characteristics of the individual member and the level of team task autonomy as a moderator variable explain differences in individual outcomes and help with predicting the effectiveness of teamwork.

People differ in the extent to which they observe, regulate, and control the appearances of self displayed in social settings and interpersonal relationships. In [6] self-monitoring as a moderator across tasks is associated with effective emotional performance. High self-monitors reported less stress and more deep acting than low self-monitors and did not experience elevated heart rate during emotional performance. Studies indicate that high self-monitors tend to receive better performance ratings and more promotions than low self-monitors and are more likely to emerge as leaders [9].

Piedmont, Weinstein (1994) evaluated the relations between the Five Factor Model and supervisor ratings of performance [6]. In their study the rating dimensions are related to personality qualities, for example the ability to work together and the ability to cope with job stress. The supervisors using a rating form were asked to rate their employees on a 5-point scale ranging from unsatisfactory (1) to excellent (5) on 12 relevant performance items that constitute three performance scales. These scales are labelled interpersonal relations (items: communicates clearly, team player), task orientation (items: hard-working, gets things done), and adaptive capacity (items: learns and adapts ready, copes effectively with setbacks, functions well in unstructured situation).

As expected the Conscientiousness (C) factor (achievementoriented, organized) correlate with the performance ratings in a consistent way. For example, competence, striving for achievement and self-discipline appear to be salient and predict successful job performance in all the rated areas. Extraversion (E) (assertive, active, social) scores were significantly correlated with ratings on the interpersonal relations scale. Extraversion (E) is a significant predictor of job success. High scores on this factor indicate a warm, engaging, positive and orientation toward others facilitating interpersonal interactions. Persons who received high scores on the Agreeableness (A) (cooperative, sympathetic, eager to help and please others) factor not only like being with others but are also willing to engage in any social activity to attain their goals. In a team situation, individuals scoring high on Extraversion (E) and low on Straightforwardness (AST) may be perceived as leaders. Low score on Straightforwardness (AST) scale of the Agreeableness (A) factor was associated with the ability to accomplish work-related goals and to adapt to changing work conditions. The Emotional Stable (N) factor was a significant predictor of ratings concerning interpersonal relations and adaptive capacity. Hardy, emotionally stable individuals were rated by their supervisors as being able to maintain a consistent relationship with others, and able to cope with various demands associated with their job.

Summarizing these findings it can be said that the Conscien- 
tiousness (C) factor scores consistently correlate with job performance across a wide range of occupational categories. The scales of Conscientiousness (C) such as competence, achievement striving, and self-discipline are specific personality qualities that underlie job success. Low Neuroticism (N) and high Extraversion (E) scores also predict high performance.

In a previous Juhasz's (2002) longitudinal study carried out in a call centres workplace, supervisors were asked to rate their employees along two main performance factors: Cognitive performance factor (CPF) and Affective performance factor (APF) [17]. The Cognitive performance factor (CPF) contains the achievement, accuracy, software knowledge, endurance, understanding of speech, memory, good communication skill, job proficiency, trainability performance dimensions, which are associated with the employees' general mental ability and skills in work performance. The $C P F$ showed significant correlations with the Openness to experience $(\mathrm{O})$ and Extraversion $(\mathrm{E})$ personality factors. The results suggested that when the supervisors assessed the operators' everyday performance according to the CPF, they preferred those people who learn quickly, are open to new technology, have fewer problems in acquiring unfamiliar knowledge and sociable, active. These results reinforced by Molleman's [26] study in which the authors confirm that individuals who are open to experience $(\mathrm{O})$ prefer tasks that demand creativity and they enjoy experimenting with new problem-solving strategies, and they are motivated to learn and prefer work that challenges them to utilize and develop their cognitive abilities. Persons low in Openness to experience (O) hardly bear the cognitive overload and avoid new and ambiguous situations that demand creativity and offer opportunities to learn. The Agreeableness (A) factor and its scales showed negative correlation with the CPF. Operators having high score on the Agreeableness (A) factor performed worse in cognitive ability tests and also their supervisors evaluated them to a lower score in those tasks which required high cognitive capacities in everyday work.

The Affective performance factor (APF) consists of quietness, politeness, pleasant voice, adaptability, cooperation, selfdiscipline, deliberateness. All these dimensions were related to personality traits that determine social behaviour in the workplace. Contrary to findings that Conscientiousness (C) has a comprehensive validity for all professional groups and all criterion types, in Juhasz's study no significant correlation between Conscientiousness (C) personality factor and the supervisory ratings has been found. To our surprise the supervisors did not appreciate the employees who scored high on the Conscientiousness (C) factor. Conscientiousness (C) indicates the working style and attitudes such as feeling responsible for good performance and having antipathy for procrastination but a score above the average can impede the efficacy in the work setting.

In sum it seems that certain studies highlight the relationship between team attributes and team outcomes, but in only one thing they agree that the nature of the team task moderates the relationship between team attributes and team outcomes. The level of Conscientiousness $(C)$ in a team influences team functioning and outcomes. High level of Conscientiousness (C) facilitates cooperation and creates an atmosphere in which individual team members are willing to learn from each other resulting in satisfied team-mates. If the level of Conscientiousness (C) is low, no one feels responsible for a task, and team members do not stick to agreements or decision. All this can cause intragroup conflicts, stress and thus dissatisfaction. Conscientiousness (C) relates to satisfaction and learning if the team is autonomous. A high level of autonomy is necessary to make decisions concerning any kinds of work issues increasingly intensive intra-team communication and the mutual adjustment of efforts. If the team members are conscientious, they actively participate in decision making, and there is an opportunity to learn. So sharing work-related attitudes and cooperate with each other, teamwork is proceeding, contributing to satisfaction [26].

Barrick, Mount's results concerning Agreeableness (A) suggest that it is not an important predictor of job performance, even in the jobs containing a large social component (e.g., sales or managers) [2]. It appears that being courteous, trusting, straight forward and soft-hearted has a smaller impact on job performance than being talkative, active, and assertive.

The role of Openness to experience $(\mathrm{O})$ in teams using computerized technology has also been examined. A computerassisted context requires both learning proficiency and creativity to integrate different communication forms which improved the decision-making performance-team, but only when the teams were high on Openness to experience [7]. Openness to experience $(\mathrm{O})$ has been found to be a valid predictor of training proficiency. One possible explanation of these findings is that individuals who score high on these factors (e.g., intelligent, curious, broad-minded, and cultured) are more likely to have a positive attitude toward learning and experience acquisition. The key component in the success of the training program is the attitude of the individual when $\mathrm{s} /$ he enters the training program [1].

\subsubsection{Configurational approach}

The personality inputs of Input-Process-Output Model include not only mean or minimum measures of personality, but the variance of traits or the mix of different traits of the team members' personality. The fit of members to each other affects interpersonal and task process in the group.

Studies on team composition attributes have highlighted the relationship between team composition characteristics and team outcomes, but the results are inconsistent. Mainly of researchers found positive relationship between the mean level of Conscientiousness $(\mathrm{C})$ in a team and performance, and some of them did not. In general the Conscientiousness $(\mathrm{C})$ is the most consistent and universal predictor of job performance for all occupational groups and all job-related criteria [1],[15]. Certain personality traits may interact with others to result in desirable, as well as 
undesirable workplace behaviours depending on the pattern and interactions of other traits. Using the supervisory rating as a reliable variable of the workplace behaviour, those highly conscientiousness workers, those low in Agreeableness (A) were found to receive lower ratings of job performance than workers high on Agreeableness (A). Highly conscientious workers who lack interpersonal sensitivity may be ineffective, particularly in jobs requiring cooperative interchange with others [40], [2].

If all members of a team are highly conscientious, each member contributes to the team task, and this will lead to many opportunities to learn from each others. High mean level of Conscientiousness (C) facilitates cooperation, and this will create an atmosphere in which individual team members are willing to learn from teammates and will also result highly satisfied individuals. However, if the level of Conscientiousness (C) is low, no one will feel responsible for a task, and team members will not stick to agreements or decisions. Members will withhold their efforts and there will be fewer chances to learn from each other. Moreover, this will easily result in an atmosphere in which members are blaming each other for social loafing. This will cause intragroup conflicts, stress, and thus dissatisfaction.

A team that consists of stable members $(\mathrm{N})$ is more effective. Stable individuals feel more confident and less insecure while collaborating with others, and therefore they will more easily bring in their own knowledge and opinions and be more receptive to the inputs of others. This will enhance the opportunities to learn. Higher levels of Emotional stability $(\mathrm{N})$ within a team will also lead to a more relaxed atmosphere. As Barrick argued, teams with unstable people tend to demonstrate more anxiety and negative affects, which lessen the satisfaction of the individual team members [3].

Individuals who are open to experience $(\mathrm{O})$ will prefer tasks that demand creativity, and they will enjoy experimenting with new problem-solving strategies; hence, they will be motivated to learn. They will prefer work that challenges them to utilize and develop their cognitive abilities. Persons low in Openness to experience $(\mathrm{O})$ will easily bear a cognitive overload and avoid new and ambiguous situations that demand creativity and offer opportunities to learn [26].

\section{Hypotheses}

H1: Communication indicators $x$ Personality

Analysing the team communication patterns we suppose that the relationship-oriented communication indicators (RCI) have more correlations with the Extroversion (E) and Agreeableness (A) personality factors than other factors. Team process-oriented communication indicators (TCI) consist of those communication utterances related to maintain the coordination and cooperation in the team. We suppose that the Uncertainty signs in communication have positive correlation with Neuroticism (N) factor and its scales and along the Thinking communication utterances the team's communication includes signs related to cognitive mental efforts, think- ing elements that are supposed to have positive correlation with the Openness to experience $(\mathrm{O})$ personality factor. Communication utterances related to relation, politeness, motivation, using the ' $W e$ ' term, and other words indicating any affects in the teamwork are supposed to have positive correlation with the Extroversion (E) and Agreeableness (A) factors and theirs scales.

\section{H2: Team performance $x$ Personality}

Knowing the relevant role of the Conscientiousness (C) factor in the work setting, we suppose its positive influence on the Team performance; meantime we expect the controversial effects of the Neuroticism (N) and Agreeableness (A) factors.

H3: Behaviour markers: "Hard" and "Soft" skills x Personality The observable "hard" skills that are related to task-solving processes such as Professional knowledge, Problem-solving, and Comply with standards have significant correlations with Neuroticism (N) and Conscientiousness (C) factors. Otherwise the "soft" skills (Communication, Cooperation, Task load management) are supposed to have relatively stronger correlations with the Extraversion (E) and Agreeableness (A) personality factors.

\section{Methods}

««« .mine The data collection was based on 17 operator teams $(\mathrm{N}=90)$ interactions $======$ The data collection was based on 17 operator teams $(\mathrm{N}=90)$ interactions »»»>. .r1736 analysis in the Simulator Centre of a Hungarian Nuclear Power Plant (NPP). The NPP Simulator Centre is a very realistic, high fidelity tool that is widely used in training and examinations creating the required level of face-validity, to be relevant for real life situations. The Nuclear Power Plant's operator teams consist of four professional fields requiring the interaction of six members:

1) Unit shift supervisor (USS), 2) Reactor operator (ROP), 3) Turbine operator (TOP), 4) Field operator (FOP), 5) Unit Electrician (UE), and 6) Shift leader (SL).

I. Personality measurement $« \lll \ll<$.mine Each team member $(\mathrm{N}=96)$ was asked to fulfil the NEO-PI-R ======= Each team member $(\mathrm{N}=96)$ was asked to fulfil the NEO-PI-R »»»»> .r1736 [8]) personality questionnaire some days prior to the scenario. This version was translated and adopted by János Nagy and Zsófia Szirmák (ELTE, Faculty of Personality). NEO-PI-R consists of five major domains of personality, as well as the six traits or facets that define each domain [28]. (Supplement 1.)

\section{Team process-oriented communication measurement}

A Together with the instructors we chose a simulator scenario ("Failure of one turbine unit") that every team had to perform. The mean duration of the scenario is about $35 \mathrm{~min}$ utes. 
B A video recording was made of the operators' activity during the selected scenario. At the beginning of the simulation study, the instructor informed the teams about this, but they did not know exactly which of the programmed scenarios would be videotaped.

C All the recorded conversations of the operators were transcribed in chronological order, identifying the operators' verbal utterances by two independent expert raters. This study exclusively focuses on those communication utterances that are likely to be related to relationship-oriented communication in the team and their influence on the team atmosphere stemming from the individual personality. About the technical communication utterances and their influence on the team performance can be read in the Soos Julia and Juhasz Marta's article in this edition (Capturing team performance differences through communication based analyses of team cognition).

III. Team performance measurement After having seen every scenario, the team as a unit performance was evaluated by instructors applying a 3-point Likert scale (1: poor; 2 : average; 3: high).

IV. Behaviour markers measurement After every scenario the instructors were asked to evaluate each individual's skills according to task-relevant ("hard") skills (Professional knowledge, Problem-solving, Comply with standards) and team-relevant ("soft") skills (Communication, Cooperation, Task load management) using a 4-point Likert scale (1: weak, 2: acceptable, 3: good, 4: excellent). These markers reflect every day used terminology in the teamwork and these skills are at the behaviour level directly observable.

The evaluation sheet can be seen in Supplement 2.

\section{Results}

6.1 Descriptive results about the team process-oriented communication indicators

Fig. 1 1 shows the most frequently used team-oriented communication utterances, which are not strongly attached to the task accomplishment but inform us about the team process and interactions during the scenario. Analysing transcribes in above mentioned way the following non-technical communication dimensions have been identified:

Uncertainty (U): Sign of a general expression of uncertainty in the conversation such as "maybe..". "I don't know exactly", "We could ask him...".

Relation (R): The principle features of team work in high risk environment are the relation-related utterances, maintenance of contact, relationship, and vigilance in sentences, like "Hold the line please!", naming the addressee, etc.

Politeness (P): The speaker gives a command, information, question or affirmation formulated politely, including formulates such as "thank you”, "Would you be so kind...", “Do it, please”. This communication form determines the team atmosphere, and indicates the mutual respect among team members.

Motivation (M): Encouragement, formulated as reinforcement, completed with motivation, stimulation. For example "It's perfect, just go on!"

First person plural (We): The speaker uses first person plural, expressed frequently in the form of "we, our, us, let's".

Affection (A): Words describing emotions, someone's emotional status, indicating astonishment, exasperation, frustration, excitement, relieve happiness or contentment. For example "I regret it", "I'm quite happy" or laughing.

Thinking, cognitive (T): Words indicating cognitive process. For example „I think...”, „Attention!”, „If... than...”, “Check it!”. These utterances may suggest problem-solving mechanism and can increase especially in facing with technical troubles. It shows how the teams are willing to endeavour the problem-solving mechanism.

All these supporting communication maintains the relationship in the team and through these non-technical communication utterances stimulate attention to each other, motivating attentive behaviour, increasing team cohesion.

As it can be seen on Fig. 1 the Thinking dimension is the most frequently used communication type indicating the welldetected mental effort during the scenario. It really rarely used the Motivation communication form in this professional teams' communication. Significantly used the first person plural (We), the relation (Relation) and emerged the uncertainty (U) communication form, too.

Fig. 2 shows the descriptive statistics of the team processoriented communication utterances according to observed teams' roles. It gives an overview of the most frequently used team- or relationship-oriented communication dimensions along different roles.

To our expectation the Unit shift supervisor (USS) is the most active team member. The USS assumed the role of speaker more often, used more frequently relation-related utterances $(\mathrm{R}, \mathrm{We}$, $\mathrm{T})$, than the other members of the team. Indicating linkage between specific features of communication and team roles linked behaviour to successfully establish the team. On the other hand, it must be mentioned that the emphasized role of Electrician (UE) in the electrician field is due to the special context of the scenario.

\subsection{Relationship between team process-oriented commu- nication and personality traits}

Table 1 shows only the significant correlations between the frequency of different types of communication utterances and the NEO-PI-R factors and scales. 


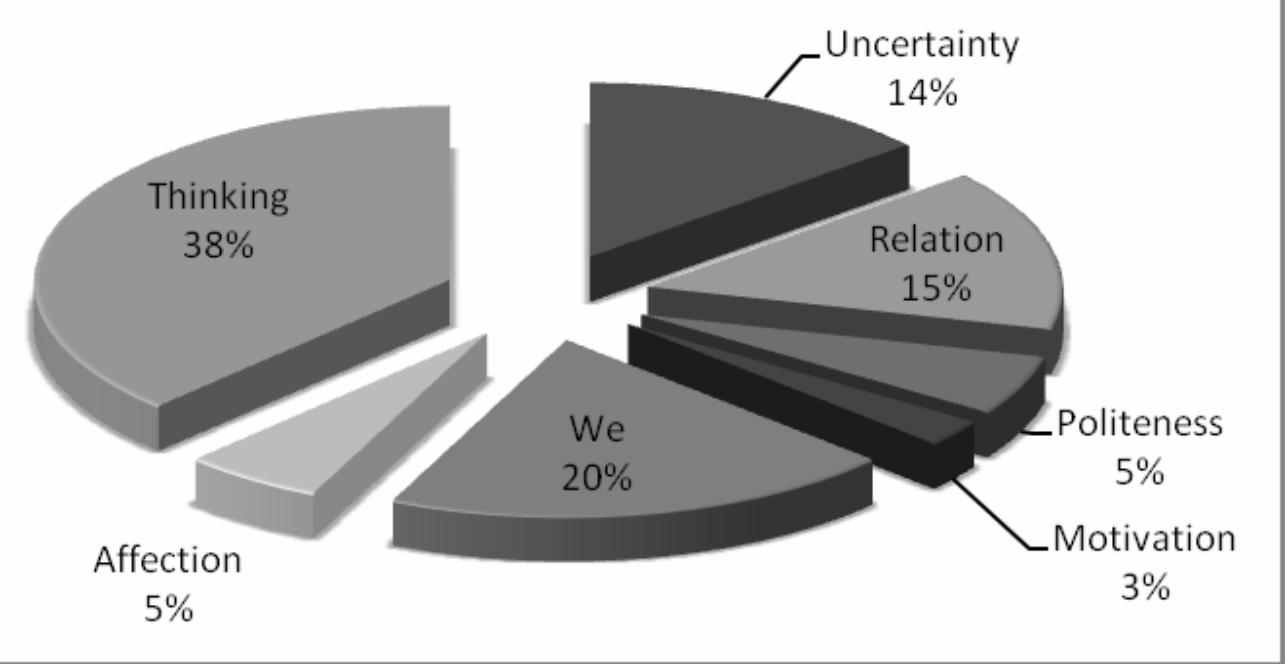

Fig. 1. Percentage of the relationship-oriented communication utterances (17 teams=N: 90$)$

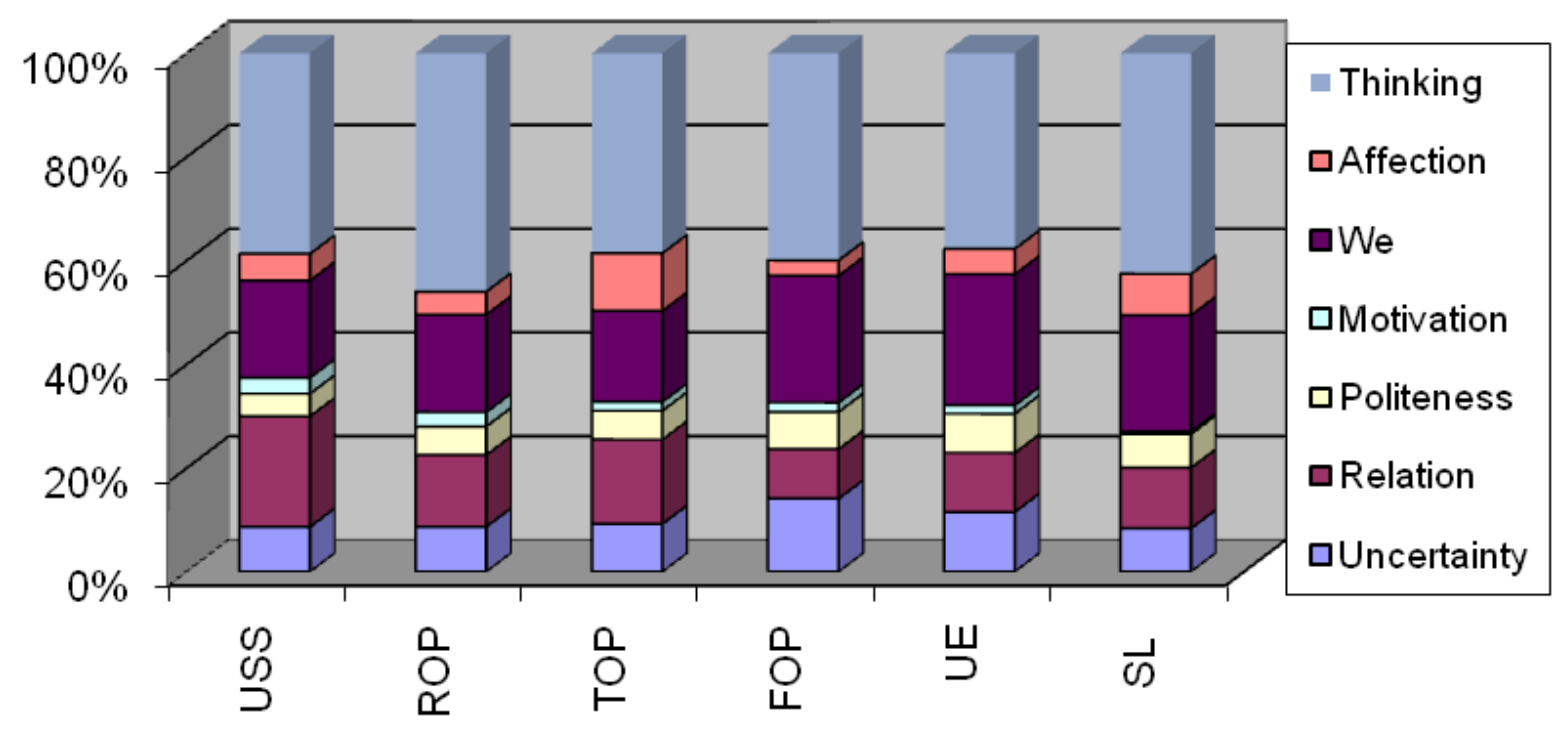

Fig. 2. Descriptive statistics of the non technical-related communication utterances according to observed teams' roles

In contrast to our expectation Neuroticism' Impulsiveness (NIM) scale shows negative correlation with Uncertainty communication mode. It means that in the impulsive operators' communication can be identified more sure, less doubts and assertive communication utterance. It seems that in highly disciplined environment the individual impulsiveness can function as a resource to combat with problems under stress.

Correlation coefficients between personality and communication indicators organize around the Extroversion (E), Agreeableness (A), Openness to experience (O) and the Relation, Politeness communication indicators. According to our expectation the Extroversion (E) and the Agreeableness (A) factors and their scales (EAS, EAC, EEX, AAL, AMO, AST) have significant relationship with the team process-oriented communication utterances (Relation and Politeness), but to our surprise the Agreeableness (A) personality factor shows negative correlations with most of these communication indicators. It seems that the higher score on the Agreeable factor and its diverse scales (AMO, AST, ACO), the lower is the possibility to use communication related to maintaining interaction in this highly task-oriented teams (Table 1 .

Politeness communication indicator has a lot of correlation co-efficiencies with various personality traits. Behind a polite communication there is a positive and open personality who is able to create an open and sincere relationship with other people and has a power to form an acceptable team ambience in which everybody respects and tolerates each other without being exaggerated kind and compliant. The Extroversion (E) and the Openness to experience $(\mathrm{O})$ factors and their scales (EAC, EEX, OFA, OFE) together affect this kind of communication in the teams. Having high score on the Achievement striving scale (CAS) and on Openness to fantasy (OFA) and to various Feelings (OFE) can help to establish a good mood and cooperation in the expert team where high performance is crucial. 
Tab. 1. Pearson correlations between personality traits and communication indicators $(* \mathrm{p}<0.05 ; * * \mathrm{p}<0.00)$

\begin{tabular}{|c|c|c|c|c|c|}
\hline \multirow[b]{2}{*}{ NEOPIR $\mathrm{N}=73$} & \multicolumn{5}{|c|}{ Communication indicators } \\
\hline & Uncertainty & Relation & Politeness & We & Thinking \\
\hline NIM Impulsiveness & $-0.28^{*}$ & & & & \\
\hline EAS Assertiveness & & $0.23^{*}$ & & & \\
\hline EAC Activity & & & $0.32^{* *}$ & & \\
\hline EEX Excitement seeking & & & $0.34^{\star *}$ & & \\
\hline E Extroversion & & & $0.34^{* *}$ & & \\
\hline OFA Fantasy & & & $0.32^{* *}$ & & \\
\hline OFE Feeling & & & $0.32^{* *}$ & & \\
\hline O Openness to experience & & & $0.26^{*}$ & & \\
\hline AAL Altruism & & $-0.29^{*}$ & & & \\
\hline AMO Modesty & & $-0.40^{\star *}$ & $-0.27^{*}$ & & $-0.26^{\star}$ \\
\hline AST Straightforwardness & & $-0.38^{* *}$ & $-0.40^{\star *}$ & $-0.24^{*}$ & $-0.29^{\star}$ \\
\hline ACO Compliance & & & $-0.35^{\star \star}$ & & $-0.27^{\star}$ \\
\hline A Agreeableness & & $-0.40^{* *}$ & $-0.31^{\star *}$ & & $-0.31^{* *}$ \\
\hline CAS Achievement striving & & & $0.27^{*}$ & & \\
\hline
\end{tabular}

For maintaining good relationship and a strong cohesion in these type of expert teams it is important to be assertive (EAS) and it seems to be lesser agreeable (A) or compliant (ACO). Opposite to our expectation the agreeable character is lesser fitting to teams operating in high risk and strongly standardised environment. Highly modest (AMO), altruist (AAL), compliant (ACO) operators are less willing to initiate new social action and easily becomes pressed by others in the team. They much less frequently name the addressee or initiate any kind of interaction during teamwork.

Four of the six scales of the Agreeableness (A) factor indicate negative correlation with Thinking communication utterances. It means that less agreeable people (A) more frequently apply expressions related to problem-solving procedure like 'think', 'attention', 'if... than' than those high score on Agreeableness.

\subsection{Team performance and personality traits}

As an output of the team process the instructors evaluated every team as a $« \ll \ll<$.mine $« \ll \ll<$.mine unit after every scenario. $17,3 \%$ of the examined 17 teams $(\mathrm{N}=90)$ were assessed as poor performance teams, $39,8 \%$ as a medium performance and $34.7 \%$ as excellent performance teams accordingly how they accomplished $=======$ unit after every scenario. $17.3 \%$ of the examined 17 teams $(\mathrm{N}=90)$ were $=======$ unit after every scenario. $17.3 \%$ of the examined 17 teams $(\mathrm{N}=90)$ were $» » »>>$ .r1736 assessed as poor performance teams, $39.8 \%$ as a medium performance and $34.7 \%$ as excellent performance teams accordingly how they accomplished »»»> .r1732 the given task, how fast and in what degree they distort from the optimal solution.

The results of regression analysis are presented in Table 2 As shown, the relevant personality traits are significantly related to team performance as dependent variable: Extraversion (E) and Conscientiousness (C). The standardized Beta Coefficients give a measure of the contribution of each variable to the model. $\Delta \mathrm{R}^{2}$ value tells that the Order scale (COR) model accounts for
$9.8 \%$ of variance in the scores. Seeing that $t$ value in this case is almost 3 , it suggests that the Order scale as a predictor variable has a moderate impact on the criterion variable, on group performance. These findings underline our hypotheses 2 and reinforce the relevant role of the Conscientiousness (C) in the wok-setting performance.

Furthermore we analysed how the homogeneity and heterogeneity of a certain personality factor alters the team performance. Previously used Levene test rejected the homogeneity of variances, the Welsch D test on Agreeableness $« \ll \ll<$.mine «««<.mine shows significant main effect on standard deviation (SD) $(\mathrm{d} 2=6.218 ; \mathrm{p}<0.05)$. So, high performed teams have greater standard deviation of the $=======$ shows significant main effect on standard deviation $(\mathrm{SD})(d 2=6.218 ; \mathrm{p}<0.05)$. So, high performed teams have greater standard deviation of the $======$ shows significant main effect on standard deviation $(\mathrm{SD})(d 2=6.218 ; p<0.05)$. So, high performed teams have greater standard deviation of the »»»»> .r1736 »»»»> .r1732 Agreeableness personality factor than poor or average performed teams.

\subsection{Behaviour markers ("soft" and "hard" skills) and person- ality traits}

After the scenario, instructors evaluated the above-mentioned team performance and the operators" "soft" and "hard" skills based on their observable behaviours. Task-related "hard" skills (Professional knowledge, Problem-solving, Comply with standard) and team-related "soft" skills (Communication, Cooperation, Task load management) were evaluated using a 4-point Likert scale (Supplement 2).

Using the stepwise lineal regression analysis from all the predictors only the Anxiety (NAN) personality trait predicts well the Professional knowledge as a $« \ll \ll<$.mine $« \ll \ll<$.mine dependent variable $(\beta=0.34 ; \mathrm{t}=3.07 ; \mathrm{p}<0.00)$ along the supervisor ratings. $======$ dependent variable $(\beta=0.34$; 
Tab. 2. Regression results for testing Team performance and various personality factors and scales.

\begin{tabular}{lllll}
\hline & \multicolumn{3}{c}{ Team performance rating (as dependent, criterion variables) } \\
\cline { 2 - 5 } Personality factors and scales (as predictor variables) & $\Delta R^{2}$ & $\beta$ & $t$ & $p$ \\
\hline Extraversion: Assertiveness (EAS) & $.048^{*}$ & .248 & 2.156 & .035 \\
Extraversion: Activity (EAC) & $.050^{*}$ & .252 & 2.190 & .032 \\
C_Conscientiousness & $.071^{*}$ & .290 & 2.552 & .013 \\
CCO_Competence & $.050^{*}$ & .252 & 2.195 & .031 \\
COR_Order & $.098^{* *}$ & .332 & 2.966 & .004 \\
CAS_Achievement striving & $.076^{*}$ & .298 & 2.633 & .010 \\
CSD_Self discipline & $.036^{*}$ & .223 & 1.923 & .058 \\
\hline
\end{tabular}

Note: ${ }^{*} \mathrm{p}<0.05$; ${ }^{* \star} \mathrm{p}<0.00$ (one-tailed), for $t$ values (for unstandardized regression coefficients) or $F$ values (for overall model). $\beta=$ Standardized Coefficients.

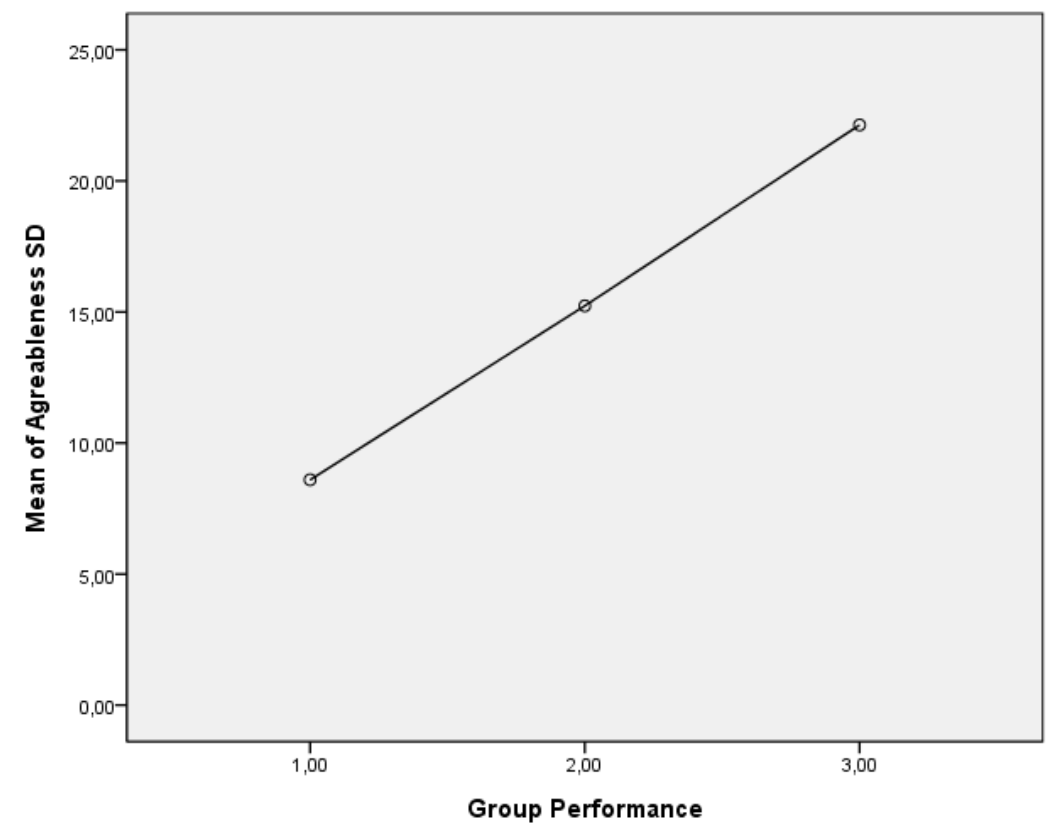

Fig. 3. Team performance ( $1=$ poor; $2=$ average; $3=$ high $)$ and Standard Deviance of Agreeableness

$t=3.07 ; p<0.00)$ along the supervisor ratings. $=======$ dependent variable $(\beta=0.34 ; t=3.07 ; p<0.00)$ along the supervisor ratings. $» » »>$. .r1736 »»»»>.r1732 The NAN left in the model even if the dependent variable has been changed: $\ll \ll \ll<$.mine $« \ll \ll<$.mine Comply with standard (keeping rules) $(\beta=0.3 ; \mathrm{t}=2.59 ; \mathrm{p}<0.05)$, Communication $(\beta=0.38$; $\mathrm{t}=3.38 ; \mathrm{p}<0.00)$ or the Cooperation $(\beta=0.37 ; \mathrm{t}=3.24$; $\mathrm{p}<0.00$ ). $=======$ Comply with standard (keeping rules) $(\beta=0.3 ; t=2.59 ; p<0.05)$, Communication $(\beta=0.38$; $t=3.38 ; p<0.00)$ or the Cooperation $(\beta=0.37 ; \mathrm{t}=3.24$; $\mathrm{p}<0.00$ ). $=======$ Comply with standard (keeping rules) ( $\beta=0.3 ; t=2.59 ; p<0.05)$, Communication $(\beta=0.38$; $t=3.38 ; p<0.00)$ or the Cooperation $(\beta=0.37 ; t=3.24$; $p<0.00) . » » »>$.r1736 »»»> .r1732

The other determinative personality trait that plays an important role in the instructors' judgment is the Conscientiousness (C) factor, precisely Dutifulness (CDU) and the Order (COR) scales. When the Comply with standard has been evaluated «««<.mine «««< personality traits $(\beta=0.251 ; \mathrm{t}=2.16 ; \mathrm{p}<0.05)$, and when the Cooperation was the dependent variable the Order (COR) personality trait $(\beta=0.31 ; \mathrm{t}=2.78 ; \mathrm{p}<0.00)$ influenced mostly the $=======$ the Dutifulness (CDU) emerged from all personality traits $(\beta=0.251 ; t=2.16 ;=======$ the Dutifulness (CDU) emerged from all personality traits $(\beta=0.251$; $t=2.16 ; » » »>>$.r1736 $\mathrm{p}<0.05)$, and when the Cooperation was the dependent variable the Order (COR) personality trait $(\beta=0.31 ; t=2.78 ; p<0.00)$ influenced mostly the »»»>> .r1732 instructors' rating. Furthermore in view of instructors an operator's communication skill mainly depends on his Assertively personality (EAS) type $« \ll \ll<$.mine $« \ll \ll<$.mine $(\beta=0.30 ; \mathrm{t}=2.62 ; \mathrm{p}<0.05) .=======(\beta=0.30 ; t=2.62 ;$ $p<0.05) .=======(\beta=0.30 ; t=2.62 ; p<0.05) . » » »>$ $. r 1736 » \gg \gg>>.11732$

Whilst the communication utterances have a strong relationship with the Extraversion (E), Openness to experience (O) and the Agreeableness (A) factors, the "soft" and "hard" skills show significant correlation only with the Neuroticism (NAN) and the 
Conscientiousness (COR, CDU) factors' scales. Our hypothesis about relationship between Extroversion (E) and Agreeableness (A) personality factors and the observable "hard" and "soft" skills has not been proved except the Assertiveness (EAS) scale.

Regarding to our findings the Neuroticism (N) factor associating with the Consciousness $(\mathrm{C})$ factor and their scales indicate their beneficial impact on the Professional knowledge, Comply with standard and on the interactive behaviour forms such as Communication and Cooperation. A bit anxiety interacting with conscientiousness (C) can help persons to form a good impression about their skills and their behaviour. These persons endeavour to be accepted by others and strive to mark out from their environment with their remarkable performance. These people react to the changing of their environment in a very sensitive way.

\section{Conclusions}

The communication of NPP operator teams reflects the environment in which the teams work. Members being together as an expert team in order to make the Plant in function must follow strictly the rules and the protocols. The situation teams are in can be characterised as a low autonomy in which each team member has their own tasks to fulfil in a regular way. Autonomy relates to the extent to which the external environment constrains a person's freedom to behave in idiosyncratic ways. In strong situations, the organization exerts considerable pressure or demands to induce conformity. These controlling forces press the individual to behave in a specific way or exhibit a very narrow range of behaviours. Controversially, in weak situations the individual determines which behaviours to undertake. So, the magnitude of the relationship between personality traits and behaviour is greater in weak situations or settings where people can perform their jobs in idiosyncratic ways.

However the major part of the operator teams' communication related to technical problem-solving, in this article we focus on the relationship or team process-oriented communication utterances are related to the maintenance relationship, initiation of an interaction, motivation of others, used in first person plural in the team.

Our study reveals that team process-oriented communication utterances first of all highly correlate with Extroversion (E) and Openness to experience $(\mathrm{O})$ personality factors, and to our surprise, in a negative direction with the Agreeableness (A) factor. In those teams these types of communication utterances are more frequent where the persons are more sociable, assertive, active and open to different feelings or fantasies but less straightforward, altruist or compliant. Similar findings were found in the Barrick, Mount (1993) study, in which the predictive validity of Agreeableness (A) was investigated introducing autonomy as a moderator variable. The validity of Agreeableness (A) was also higher in high-autonomy jobs compared with lowautonomy ones, but the correlation was negative. These findings suggest that the degree of the job autonomy influences the va- lidity of personality dimensions. It means that in NPP operator teams in that members work in high autonomy, so-called strong situation in which their personality traits are not permit to be evolved. Even if they are working together as a team it seems that Agreeableness (A) softly impedes the effective team functioning. Communication utterances related to problem-solving (Thinking) have a negative correlation with the Agreeableness (A) personality factor, too. This finding underlines Juhasz's (2005) preview study in which the Cognitive Performance Factor had a strong negative correlation with the Agreeableness (A) personality factor.

During the team-process the operators" "soft" and "hard" skills have remarkable relationship with the personality traits. First of all Professional knowledge and Coordination behaviour markers show significant correlations with Neuroticism $(\mathrm{N})$ and Conscientiousness (C) personality factors. The stable role of Conscientiousness $(\mathrm{C})$ has been reinforced, precisely the Dutifulness (CDU) and the Order (COR) that mainly influence the operators' Keeping rules and Cooperation skills that largely determine their behaviour in this type of work setting. It seems that Team-performance as a team process output is directly influenced by the Conscientiousness (C) and the Extraversion (E) personality factors based on instructors' evaluations.

\section{References}

1 Barrick M, Mount M, The Big Five Personality Dimensions and Job Performance: A Meta Analysis, Personnel Psychology, 44, (1991.), 1-26.

2 _ Autonomy as a Moderator of the Relationship Between the Big Five Personality Dimensions and job Performance, Journal of Applied Psychology, 78(1), (1993.), 111-118.

3 Barrick M, Stewart G, Neubert M, Mount M, Relating member ability and personality to work-team processes and team effectiveness, Journal of Applied Psychology, 83(3), (1998.), 377-391.

4 Barry B, Stewart G, Composition, process, and performance in selfmanaged groups: The role of personality, Journal of Applied Psychology, 82, (1997.), 62-78.

5 Beaty J, Cleveland J, Murphy K, The Relation Between Personality and Contextual Performance in ,Strong” Versus ,Weak” Situation, Human Performance, 14(2), (2001.), 125-148.

6 Bono J, Vey M, Personality and Emotional Performance: Extraversion, Neuroticism, and Self-Monitoring, Journal of Occupational Health Psychology, 12(2), (2007.), 177-192.

7 Colquitt J, Lepine J, Hollenbeck J, Ilgen D, Sheppard L, Computerassisted communication and team decision-making performance: The moderating effect of Openness to experience, Journal of Occupational Health Psychology, 87(2), (2002.), 402-410.

8 Costa P, McCrae R, Revised NEO Personality Inventory (NEO-PI-R) and NEO Five-Factor Inventory (NEO-FFI), Psychological Assessment Resources, Odessa, 1992.

9 Day D, Unckless A, Schleicher D, Hiller N, Self-Monitoring Personality at Work: A Meta-Analytic Investigation of Construct Validity, Journal of Applied Psychology, 87(2), (2002.), 390-401.

10 Dietrich R, Grommes P, Neuper S, Language processing, Group interaction in high risk environments: The GIHRE Project (Dietrich R, Childress T, eds.), Ashgate, Burlington, 2004.

11 Diegritz T, Fürst C, Empirische Sprechhandlungsforschung: Ansätze zur 
Analyse und Typisierung authentischer Äußerungen, Univ. Bilbiothek., Erlangen, 1999., ISBN 3930357291.

12 Fukuda R, Voggenberger T, Sträter O, Bubb H, Analysis of communication in nuclear power plants, Quality of work and products in enterprises of the future: Proceedings of the Annual Spring Conference of the GfA on the occasion of the 50th Anniversary of the Foundation of the Gesellschaft für Arbeitswissenschaft (Strasser H, Kluth K, Rausch H, Bubb H, eds.), Ergonomia, Stuttgart, 2003., 615-618.

13 Furnham A, Personality and Organization: A European perspective on Personality Assessment in Organizations, Personality and organization (Scheinder B, Smith D B, eds.), Lawrence Erlbaum Associates, 2004.

14 Gellatly I R, Irving P G, Personality, autonomy, and contextual performance of managers, Human Performance, 14(3), (2001), 231-245.

15 Hogan J, Ones D, Conscientiousness and Integrity at Work, The Five-Factor Model of personality (Wiggins J, ed.), The Guilford Press, New York, 1997.

16 Hutchby I, Wooffitt R, Conversation Analysis, Polity Press, Cambridge, 1998., ISBN 074561549X.

17 Juhász M, Application of Five Factor Model in the personnel selection and the performance rating: Influence the criteria changes on the predictive validity and supervisory rating, Ph.D. Thesis, BME-ELTE, Budapest, 2002.

18 _ Influence of criterion change on supervisory rating concerning the Five-Factor Model, Periodica Polytechnica, 13(1), (2005.), 45-59, http:// www.pp.bme.hu/so/index.html

19 Krifka M, Martens S, Schwarz F, Group Interaction in the Cockpit: Some Linguistic Factors, Linguistische Berichte, 12(Special Issue), (2003), 75101.

20 Krifka M, Structural features of language and language use, Group interaction in high risk environments: The GIHRE Project (Dietrich R, Childress T M, eds.), Ashgate, Burlington, 2004.

21 LePine J A, Hollenbeck J R, Ilgen D R, Hedlund J, Effects of individual differences on the performance of hierarchical decision-making teams: Much more than g., Journal of Applied Psychology, 82, (1997), 803-811.

22 Littlepage G, Schmidt G, Whisler E, Frost A, An input-process-output analysis of influence and performance in problem-solving groups, Journal of Personality and Social Psychology, 69, (1995.), 877-889.

23 Martens S, Schwarz F, Linguistic factor, Group interaction in high risk environments: The GIHRE Project (Dietrich R, Childress T, eds.), Ashgate, Burlington, 2004

24 McCrae R, Costa P, The Structure of Interpersonal Traits: Wiggins's Circumplex and the Five-Factor Model, Journal of Personality and Social Psychology, 56(4), (1989.), 586-595.

25 Frese M, The changing nature of work, Work and Organisational Psychology (Chmiel N, ed.), Blackwell Published, Oxford, 2000.

26 Molleman E, Nauta A, Jehn K, Person-job fit applied to teamwork: a multilevel approach, Small Group Research, 35, (2004.), 515-539.

27 Moynihan L, Peterson R, The role of personality in group process, Personality and organization (Scheinder B, Smith D, eds.), Lawrence Erlbaum Associates, 2004., 2004.

28 De Fruyt F, McCrae R, Szirmák Z, Nagy J, The Five-Factor Personality Inventory as a measure of the Five-Factor Model: Belgian, American, and Hungarian comparisons with the NEO-PI-R, Assessment, 11, (2004.), 207215.

29 Pennebaker J, King L, Linguistic styles: Language use as an individual difference, Journal of Personality and Social Psychology, 77, (1999.), 12961312.

30 Piedmont R, Weinstein H, Predicting Supervisor Rating of job performance Using the NEO Personality Inventory, Journal of Psychology, 128, (1994.), 255-265.

31 Sacks H, Lectures on Conversation, Basil Blackwell, Oxford, 1992., ISBN 1557862192 .
32 Salas E, Sims D, Burke C, Is there a „Big Five” in Teamwork?, Small Group Research, 36, (2005.), 555-599.

33 Searle J, Speech Acts: An essay in the philosophy of language, Cambridge University Press, 1968., ISBN 051209626X.

34 Sexton J, Helmreich R, Analyzing cockpit communication: The links between language, performance, error and workload, The Ohio State University, Columbus, $\mathrm{OH}$.

35 _ Analyzing cockpit communication: The links between language, performance, error and workload, Human Performance in Extreme Environments, 5, (2000.), 63-68.

36 Stewart G, Barrick M, Four lessons learned from the person-situation debate: A review and research agenda, Personality and organization (Scheinder B, Smith D, eds.), Lawrence Erlbaum Associates, 2004., 2004.

37 Sträter O, Group Interaction in High Risk Environments: Communication in NPP, GRS Report(A-3020), (2002.)

38 Sträter O, Fokuda R, Communication in Nuclear Power Plants (NPP), Group interaction in high risk environments: The GIHRE Project (Dietrich R, Childress T, eds.), Ashgate, Burlington, 2004., 55-73.

39 Waung M, Brice T, The effects of conscientiousness and opportunity to caucus on group performance, Small Group Research, 29, (1998.), 624-634.

40 Witt L, Barrick M, Burke L, Mount M, Research reports: The interactive effects of Conscientiousness and Agreeableness on job performance, Journal of Applied Psychology, 87(1), (2002.), 164-169.

41 Wiggins J (ed.), The Five-Factor Model of personality, Guilford, New York, 1996, ISBN 9781572300682.

42 Comer D, A model of Social Loafing in Real Work Groups. Human Factors, The Journal of the Human Factors and Ergonomics Society, 51, (April 1995), 115-125.

43 Earley P, Social Loafting and Collectivism: A Comparison of the United States and People's Republic of China, Administrative Science Quarterly, 34(4), (Dec. 1989), 565-581.

$44 \ldots$, East Meets Mideast Further Explorations of Collectivistic and Individualistic Work Groups, The Academy and Management Journal, 36(2), (Apr 1993), 319-348.

45 Wagner $\mathrm{J}$.

46 Weick K E, Penner D D, Discrepant membership as an occasion for effective cooperation, Sociometry, (1969). 


\section{Supplement 1: NEO-PI-R factors and scales}

Neuroticism N; Anxiety NAN; Angry hostility NAH; Depression NDE; Self consciousness NSC; Impulsiveness NIM; Vulnerability NVU.

Extroversion E; Warmth EWA; Gregariousness EGR; Assertiveness EAS; Activity EAC; Excitement seeking EEX; Positive emotions EPE.

Openness to experience O; Fantasy OFA; Aesthetics OAE; Feeling OFE; Actions OAC; Ideas OID; Values OVA.

Agreeableness A; Trust ATR; Straightforwardness AST; Altruism AAL; Compliance ACO; Modesty AMO; Tender mindedness ATM.

Conscientiousness C; Competence CCO; Order COR; Dutifulness CDU; Striving for achievement CAS; Self discipline CSD; Deliberation CDL. 
Supplement 2: Evaluating Sheet about the personnel observable Behaviour

Behaviour markers

\begin{tabular}{cccc}
\multicolumn{2}{c}{ Instructor's name: } & Date: & Code: \\
\hline $\mathbf{1}$ & $\mathbf{2}$ & $\mathbf{3}$ & $\mathbf{4}$ \\
\hline Weak & Acceptable & Good & Excellent
\end{tabular}

\begin{tabular}{|c|c|c|c|c|c|c|c|}
\hline Roles & Name & $\begin{array}{l}\text { Professional } \\
\text { knowledge }\end{array}$ & $\begin{array}{l}\text { Problem- } \\
\text { solving }\end{array}$ & $\begin{array}{l}\text { Keep the } \\
\text { rules (com- } \\
\text { ply with } \\
\text { standard) }\end{array}$ & $\begin{array}{l}\text { Communi- } \\
\text { cation }\end{array}$ & $\begin{array}{l}\text { Cooper- } \\
\text { ation }\end{array}$ & $\begin{array}{l}\text { Task load } \\
\text { manage- } \\
\text { ment }\end{array}$ \\
\hline
\end{tabular}

Unit shift supervisor (USS)

Turbine operator (TOP)

Field operator (FOP)

Reactor operator (ROP)

Unit Electrician (UE)

Shift leader (SL)

Impression about the whole team performance:

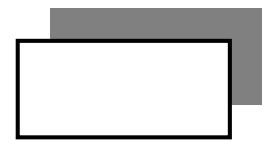

\begin{tabular}{ccc}
\hline 1 & 2 & 3 \\
\hline Poor & Average & High \\
\hline
\end{tabular}

